\title{
Erratum to: Hydrogen isotope replacement changes hydration and large scale structure, but not small scale structure, of agarose hydrogel networks
}

Tom Brenner ${ }^{1, a}$, Rando Tuvikene ${ }^{2}$, Yiping $\mathrm{Cao}^{3}$, Yapeng Fang ${ }^{3}$, Masahiro Rikukawa ${ }^{1}$, William S. Price ${ }^{4}$, and Shingo Matsukawa ${ }^{5}$

1 Faculty of Science and Technology, Materials and Life Sciences, Sophia University, 7-1 Kioicho, Chiyoda, Tokyo 102-8554, Japan

2 School of Natural Sciences and Health, Tallinn University, Narva mnt 29, 10120 Tallinn, Estonia

3 Glyn O. Phillips Hydrocolloids Research Centre, School of Food and Pharmaceutical Engineering, Faculty of Light Industry, Hubei University of Technology, Wuchang, Wuhan 430068, China

4 Nanoscale Organisation and Dynamics Group, School of Science and Health, Western Sydney University, Penrith, NSW 2751, Australia

${ }^{5}$ Graduate School of Science and Technology, Tokyo University of Marine Science and Technology, 4-5-7 Konan, Minato-Ku, Tokyo 108-8477, Japan

Original article: Eur. Phys. J. E (2019) 42: 53, https://doi.org/10.1140/epje/i2019-11816-9

Received 1 June 2019

Published online: 23 July 2019

(c) EDP Sciences / Società Italiana di Fisica / Springer-Verlag GmbH Germany, part of Springer Nature, 2019

With reference to eq. (5) in the original article, which reads

$$
G_{\text {free }} / k_{\mathrm{b}} T=\sum_{i} W_{i} \ln \left(\frac{W_{i}}{\sum_{i} W_{i}}\right)+\sum_{i} \frac{W_{i}}{\beta} \ln \left(\frac{\sum_{i} W_{i}}{\sum_{i} W_{i}+\beta W_{\mathrm{Ag}}}\right)+W_{\mathrm{Ag}} \ln \left(\frac{\beta W_{\mathrm{Ag}}}{\sum_{i} W_{i}+\beta W_{\mathrm{Ag}}}\right)+\beta W_{\mathrm{Ag}} \sum_{i} C_{i} \omega_{i}
$$

we have neglected to state that the $C_{i}$ terms refer to volume fractions (of the three water isotopomers within the gel phase).

We apologize to the readers for any inconvenience.

\footnotetext{
${ }^{a}$ e-mail: physicalchemistrytom@gmail.com (corresponding author)
} 